\section{Skin-deep target}

\section{By Michael J. Haas, Senior Writer}

There are at least a dozen approved drugs for eczema, but none for long-term or continuous treatment of the condition. First-line drugs such as corticosteroids cannot be used for more than a few consecutive weeks because of potential skin thinning. Second-line treatments such as topical calcineurin inhibitors carry black-box warnings of an increased cancer risk.

Now, U.S. researchers have found a new target for atopic dermatitisthymic stromal lymphopoietin (TSLP). ${ }^{1}$ Allergy researchers contacted by SciBX agreed that inhibition of TSLP may be a potential new strategy to treat atopic dermatitis and other atopic allergic diseases. Indeed, recent papers have elucidated a role for TSLP in asthma and chronic obstructive pulmonary disease $(\mathrm{COPD})^{2}$ and have suggested a broader role for the protein in regulating inflammatory and allergic responses. ${ }^{3,4}$

At the same time, this potentially broader role for TSLP leaves an open question as to how it affects immune responses beyond atopic aller-
"The holy grail of atopic dermatitis therapy is an agent that could be used safely over the long term, especially as a prophylaxis."

- Charl van Zyl, Jado Technologies GmbH significantly less skin inflammation and lower levels of IL-4 and IL-13 than wild-type mice not receiving the $\mathrm{mAb}$.

The team wrote in PNAS that their results demonstrated an essential role for TSLP in activating the production of proinflammatory cytokines in $\mathrm{T}$ cells that were recruited to the skin in response to allergic stimulation.

\section{Going long}

Neither Geha nor Rui He, a research fellow in pediatrics at Children's Hospital Boston and lead author on the PNAS paper, returned calls seeking comment. But other allergy researchers contacted by SciBX agreed that the results made a strong case for TSLP as a new target for atopic dermatitis.

Nevertheless, they said it remained to be seen whether small molecule TSLP inhibitors would be safe for long-term use.

"Atopic dermatitis is a complex disease, and there is a need for a better understanding of the pathways involved and possible intervention strategies," said Charl van Zyl, CEO of Jado Technologies GmbH. "TSLP is an interesting target within the cascade of allergic inflammation. The way the researchers have isolated and demonstrated its mechanism of action makes it attractive" for small molecule drug development, he said.

van Zyl noted that TSLP works in the affected skin to activate the final stages of the allergic inflammatory response. At first glance, he said, that might suggest that a topical TSLP inhibitor would be the right approach. "But an oral drug that is readily bioavailable in the bloodstream gies. The answer will determine whether TSLP inhibitors could become long-term therapeutics in eczema patients.

TSLP is a cytokine expressed by keratinocytes and other epithelial cells. It acts by binding a heterodimeric receptor composed of TSLP receptor (TSLPR; CLRF2) and IL-7 receptor (CD127).

In a paper in the Proceedings of the National Academy of Sciences, researchers from Harvard Medical School, Children's Hospital Boston and Virginia Mason Medical Center's Benaroya Research Institute reported that TSLP plays a role in activating allergic skin inflammation. ${ }^{1}$ The team was led by Raif Geha, professor of pediatrics at Harvard and chief of immunology at Children's Hospital Boston.

In a TSLPR knockout mouse model of allergic skin inflammation, antigen-specific $\mathrm{T}$ cells accumulated in the skin at the site of allergic stimulus but produced significantly lower levels of IL-4 and IL-13 than the $\mathrm{T}$ cells of wild-type mice $(p<0.0001)$. The reduction of these proinflammatory cytokines led to substantially less skin inflammation in the knockouts than in wild-type mice.

In vitro experiments showed that TSLP induced the production of IL-4 and IL-13 in antigen-specific T cells from normal mice but not in those from TSLPR-deficient mice, thus suggesting that the TSLPR-TSLP interaction activated the local inflammatory response.

Indeed, when the team gave normal mice an intradermal injection of an anti-TSLP $m A b$ prior to allergic stimulation of the skin, the mice had could work too, since the skin is well supplied with blood."

van Zyl noted that the eczema market lacks oral drugs that could be used as prophylactic follow-ups to topical therapy and wanted to know whether TSLP inhibition would have a better long-term safety profile than the approved drugs. "The holy grail of atopic dermatitis therapy is an agent that could be used safely over the long term, especially as a prophylaxis. Studies suggest that this approach could essentially cure the

$\mathrm{He}$ added that an oral agent would be easier for patients to take and would provide more thorough protection than the all-over application of a topical agent.

Jado is developing TF002, a small molecule that disrupts the lipid rafts in the membranes of mast cells and thus prevents IgE from binding and activating the cells. A topical formulation of the compound is in a Phase II trial for the long-term treatment of atopic dermatitis. The company expects to complete the trial in $2 \mathrm{Q} 09$.

An oral formulation of TF002 is in Phase II testing to treat antihistamine-resistant urticaria, a condition commonly known as hives.

Ying Sun, a reader in the Division of Asthma, Allergy and Lung Biology at King's College London, said the work of Geha's team "gives further support for the idea that TSLP might play a role in atopic disease, including atopic dermatitis."

She added that TSLP plays an important role in the development disease," he said. 


\section{TARGETS \& MECHANISMS}

of T helper type 2 cells (Th2 cells) but does not appear to be produced in response to a particular type of allergen or antigen. Thus, she said TSLP inhibition could be an effective strategy for treating a number of Th2 cell-mediated diseases, including asthma and atopic dermatitis.

Indeed, Sun and other researchers at King's College reported in the August Journal of Immunology that TSLP expression was higher in the airways of asthma and COPD patients than that seen in healthy individuals. $^{2}$

Sun said that antibodies-such as the anti-TSLP mAb used by Geha's team-have several disadvantages compared to small molecule drug approaches.

"Cost, inconvenience of administration by injection, long-term side effects and lower effectiveness" are common problems experienced by patients taking therapeutic antibodies, she said, citing Xolair as an example.

Xolair omalizumab, an injectable anti-IgE antibody marketed by partners Genentech Inc. and Novartis AG to treat severe allergic asthma, is associated with increased risk of anaphylaxis (severe allergic reaction) and cancer.

But Sun also thinks more information is needed before deciding if TSLP inhibition with a small molecule is a good long-term therapeutic strategy for atopic dermatitis.

"The next question to ask is how targeting TSLP systemically might affect all Th2 immune responses," she said. "There is lack of evidence to show whether or not TSLP inhibition would have a systemic effect on Th2 functions in humans."

A potential solution, Sun said, is to start with topical formulations of TSLP inhibitors, which should have fewer potential side effects than an oral agent.

van Zyl agreed. "If TSLP were found to have multiple modes of action," a topical would be a better mode of delivery.

Erik Halvorsen, director of technology and business development at Children's Hospital Boston, told SciBX that an IP evaluation of the findings reported in PNAS is pending. He declined to disclose details.

\section{REFERENCES}

1. He, R. et al. Proc. Natl. Acad. Sci. USA; published online Aug. 19, 2008; doi:10.1073/pnas.0801532105

Contact: Raif Geha, Children's Hospital, Boston, Mass. e-mail: raif.geha@childrens.harvard.edu

2. Ying, S. et al. J. Immunol. 181, 2790-2798 (2008)

3. Rochman, Y. \& Leonard, W. Curr. Opin. Pharmacol. 8, 249-254 (2008)

4. Wang, J. \& Xing, F. Cell. Mol. Immunol. 5, 99-106 (2008)

\section{COMPANIES AND INSTITUTIONS MENTIONED}

Children's Hospital Boston, Boston, Mass.

Genentech Inc. (NYSE:DNA), South San Francisco, Calif.

Harvard Medical School, Boston, Mass.

Jado Technologies GmbH, Dresden, Germany

King's College London, London, U.K.

Novartis AG (NYSE:NVS; SWX:NOVN), Basel, Switzerland

Virginia Mason Medical Center, Seattle, Wash. 